\title{
Epilepsy signals of each band analysis based on the average phase-synchronization time matrix
}

\author{
Yong Hua $\mathrm{Li}^{1}$, Jia-Fei Dai ${ }^{2}$, Jun Wang ${ }^{1, \mathrm{a}^{*}}$ and Feng-Zhen $\mathrm{Hou}^{3, \mathrm{~b}}$ \\ ${ }^{1}$ Image Processing and Image Communications Key Lab. , Nanjing Univ. of Posts \& Telecomm. , \\ Nanjing 210003, China \\ ${ }^{2}$ Nanjing General Hospital of Nanjing Military Command, Nanjing, 210002, China \\ ${ }^{3}$ School of Science, China Pharmaceutical University, Nanjing 210009, China \\ a E-mail: wangj@njupt.edu.cn, ${ }^{b}$ E-mail: houfz@cpu.edu.cn
}

\begin{abstract}
Keywords: Epilepsy, phase synchronization, APSTM, EEG
Abstract. This paper studied the epilepsy signal in different frequency bands based on the average phase-synchronization time matrix (APSTM). APSTM algorithm can probe the change in the synchrony of the underlying system with high sensitivity, and be applied to multichannel, nonlinear time series in noisy. This paper uses the EEG filter provided by the EEGLAB to extract the four common waves : theta, alpha, beta and gamma, which are from the normal and epilepsy EEG signals. The four frequency bands of EEG signals are analysed based on the APSTM algorithm, and the results show that the APSTM determinant can distinguish the epilepsy from the normal EEG signals clearly. And when APSTM determinant detects epileptic discharge ,the theta wave is the most significant, and the beta wave can only generally detect epileptic discharge, while alpha and gamma waves are ineffective.
\end{abstract}

\section{Introduction}

Epilepsy is a common brain disease, which symptom is that the brain neurons excitability can be increased, the synchronized discharge is excessive, and then will give rise to transient abnormal condition of the central nervous system function .Nowadays, about $1 \%$ people have epilepsy in the world. Because of its persistence and repeatability, epilepsy is not only harmful to the patient's physical and psychological condition, but also has a bad effect on society[1]. Therefore, the detection and prediction for the epilepsy becomes very important.

In recent years the study of epilepsy signals has become very popular, especially the research for the synchronization of epilepsy signal plays an important role. Many scholars have come up with some methods to measure the EEG synchronization, such as cross-correlation, mutual information, phase synchronization index (PSI)[2] and the average phase synchronization time (APST)[3]. These methods mostly study the synchronous relationship of the double channel. But the brain is a complex network composed by a large number of neurons, there is a mutual influence between the various channel, therefore it is not enough to analyze the relationship only for a pair of channels. Nowadays, the research for multi-channel EEG has become more and more widely. Some researchers have found that EEG synchronization is the main feature of information exchange in different brain regions, and for epilepsy, the main mechanism is the synchronization during its pathological state. Thus, researchers have proposed the simultaneous analysis of multi-channel, such as APSTM[4]. EEG is divided into five bands usually, that are delta waves $(0.5 \sim 4 \mathrm{~Hz})$, theta $(4 \sim 7 \mathrm{~Hz})$ wave, alpha wave $(8 \sim 13 \mathrm{~Hz})$, beta wave $(14 \sim 30 \mathrm{~Hz})$ and gamma wave $(30 \sim 70 \mathrm{~Hz})$. Because the EEG features will have a big difference under different conditions, the researchers analyze it according to different bands, such as the PSI pair[5]. APSTM is more sensitive than other methods that are used to measure the synchronous features of the phase, and can monitor the synchronous state of the system. This paper analyzes several bands based on APSTM, the results show that the theta wave is the most clearly, and beta can only generally detect epileptic discharge. 


\section{Methods}

APST. Given $X(t)$, it's a real signal of nonlinear oscillators system, the complex analytical signal is defined as

$$
\mathrm{V}(\mathrm{T})=\mathrm{X}(\mathrm{t})+\mathrm{i} \mathrm{X}_{\mathrm{h}}(\mathrm{t})=\mathrm{A}(\mathrm{t}) \mathrm{e}^{\Phi(\mathrm{t})}
$$

Where and $\Phi(\mathrm{t})$ stand for the instantaneous amplitude and phase of signal $\mathrm{X}(\mathrm{t})$, respectively, and $\mathrm{X}_{\mathrm{h}}(\mathrm{t})$ is the Hilbert transform of $\mathrm{X}(\mathrm{t})$, defined as

$$
X_{h}(t)=\frac{1}{\pi} P \cdot V \cdot \int_{-\infty}^{\infty} \frac{X(t)}{t-\tau} d \tau
$$

Here, P.V. stands for Cauchy principal value. Given two time series $x(t), y(t)$, which define its phase variable $\Phi_{\mathrm{i}}(\mathrm{t})$ and $\Phi_{\mathrm{j}}(\mathrm{t})$ respectively. Then, $\mathrm{x}(\mathrm{t})$ and $\mathrm{y}(\mathrm{t})$ are said to be in $\mathrm{n}: \mathrm{m}$ phase synchronization when the inequality $\Delta \Phi(\mathrm{t})=\left|\mathrm{n} \Phi_{\mathrm{i}}(\mathrm{t})-\mathrm{m} \Phi_{\mathrm{j}}(\mathrm{t})\right|<2 \pi$, where $\mathrm{n}$ and $\mathrm{m}$ are positive integers[6]. In this paper, we focus on the case of $n: m=1: 1$ phase synchronization. Due to noise and non-stationary, a phase synchronization status so defined can last for only a finite amount of time. Thus a practically useful quantity to characterize the degree of phase synchronization is APST(denoted by $\tau$ ). It is defined as $\tau=\lim _{\mathrm{x} \rightarrow \infty} \frac{1}{\mathrm{n}} \sum_{\mathrm{i}=1}^{\mathrm{n}} \Delta \mathrm{t}_{\mathrm{i}}$, where $\Delta \mathrm{t}_{\mathrm{i}}$ represents a time interval, where $\Delta \Phi(\mathrm{t})$ remains bounded within $2 \pi$. We know when are synchronized within a time interval $\Delta \mathrm{t}_{\mathrm{i},} \mathrm{i}=1,2,3, \mathrm{~K}, \mathrm{n}$, APST is the length of the time interval. For calculation purposes, we calculate APST by using a large time interval $\Delta \mathrm{T}$ observation during which a number of $2 \pi$ changes in the phase difference occur. As the system evolves, i.e., as the "window moves", the APST can change. Let $\tau_{(\mathrm{i}, \mathrm{j})}(\mathrm{t})$ stand for APST that channel between $i$ and $j$ at time $t$, where $t$ is the time at the end of a window.

Setting APSTM diagonal elements. Suppose there are $N$ channels in total, which the interaction between each channel. To take full advantage of all available data, we define this an $\mathrm{N}^{*} \mathrm{~N}$ symmetric matrix of APST for all pairs of channels. If the diagonal elements inappropriate, will cause the matrix become quite singular matrix, hampering further analysis, and therefore a criterion is needed for properly choosing the diagonal elements. Here we use a method based on random matrix theory to analyze APSTM, and find to the optimal value of the diagonal elements[4]. Let diagonal elements denote of $\alpha$, according to random matrix theory, APSTM singular area can be estimated via $\alpha=2 \sigma_{\Gamma} \sqrt{\mathrm{N}}+\left\langle\tau_{i, j}\right\rangle, \mathrm{i} \neq \mathrm{j}$, where $\sigma_{\Gamma}$ represents matrix $\Gamma$ standard deviation, $\mathrm{N}$ is the number of channel, $\left\langle\tau_{\mathrm{i}, \mathrm{j}}\right\rangle$ stands for the average value of all off-diagonal elements. This singular region should be avoided. we can set $\alpha$ as $\left\langle\tau_{i, j}\right\rangle$ multiples, and avoid $\alpha$ falling singular region of the matrix when $2 \sigma_{\Gamma} \sqrt{N}\left\langle\tau_{i, j}\right\rangle$. In this way, the variance is still appreciable so that the matrix may capture synchronous characteristic changes in the underlying system.

\section{Analysis EEG signals of each band base on APSTM}

EEG data recording and preprocessing. The EEG data is from the latest clinical collection, including epileptic and normal EEG signal, which were collected from the General Hospital of the Nanjing Military Region. The original EEG signals sample frequency is 512HZ. EEG signals are so susceptible to noise that we filter the EEG signals into $[0.1,80] \mathrm{HZ}$. In order to analyze the effect of different frequency bands for data APSTM, we choose four common waves that the theta waves $([4,8]$ 
$\mathrm{Hz})$, alpha waves $([8,12] \mathrm{Hz})$, beta waves $([12,30] \mathrm{Hz})$, and gamma waves $([30,80] \mathrm{Hz})$, which are provided by EEGLAB filters[7].

Analysis of each band EEG signal. To reduce the impact of noise, we use the differential signals from pairs of channels with no common reference. Fig.1. shows a representative example, which EEG differential signal from two channels, it indicates that (a) is the epileptic and (b) is the normal. And the original signal amplitude will reach to its larger degree when it is in the epilepsy discharge period. From the pictures, we can see that there are four distinct epileptic discharge period, it's 10-6000, 13000-22000, 26000-36000 and 38000-45000. To calculate APSTM determinant, we extract the theta waves, alpha waves, beta waves and gamma waves by the filter.
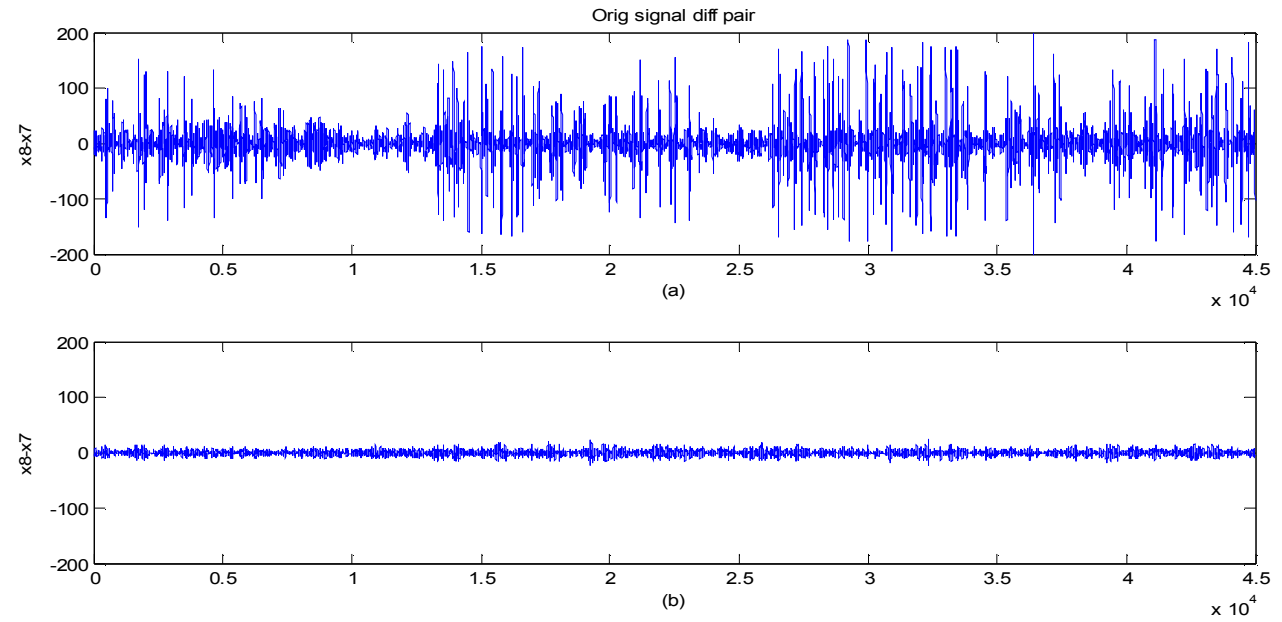

Fig. 1. (a) the epileptic EEG signal pairs (b) the normal EEG signal pairs.
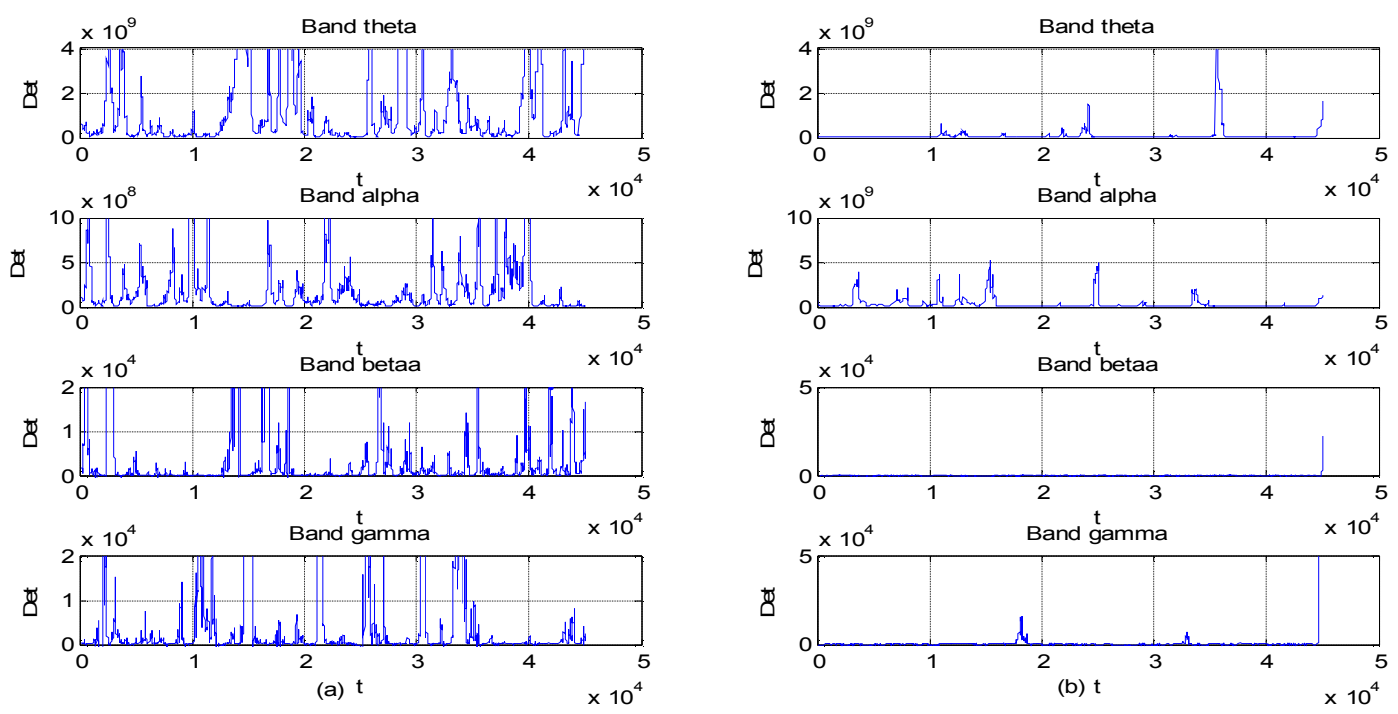

Fig. 2. (a) APSTM determinant of epileptic signal, (b) APSTM determinant of epileptic normal.

The APSTM determinant change is high during the epileptic discharge period, that is also called EEG synchronization period. Fig2(a) shows the APSTM determinant of epileptic signal varying with the time ,while Fig2(b) is the normal signal. From the Fig2(a),we can know that the theta and beta waves can detect the epileptic discharge period clearly, especially the theta wave.

But for the Fig2(b), the APSTM determinant change is low, which indicates that the degree of synchronization is very weak. In order to observe the variation of the determinant, seven bands determinant are chosen to be analyzed and quantified that are 10-6000, 6000-13000, 13000-22000, $22000-26000,26000-36000,36000-38000$ and 38000-45000, as shown in fig3. We can see that the theta waves determinant is higher during epileptic discharge when compared with its stopping 


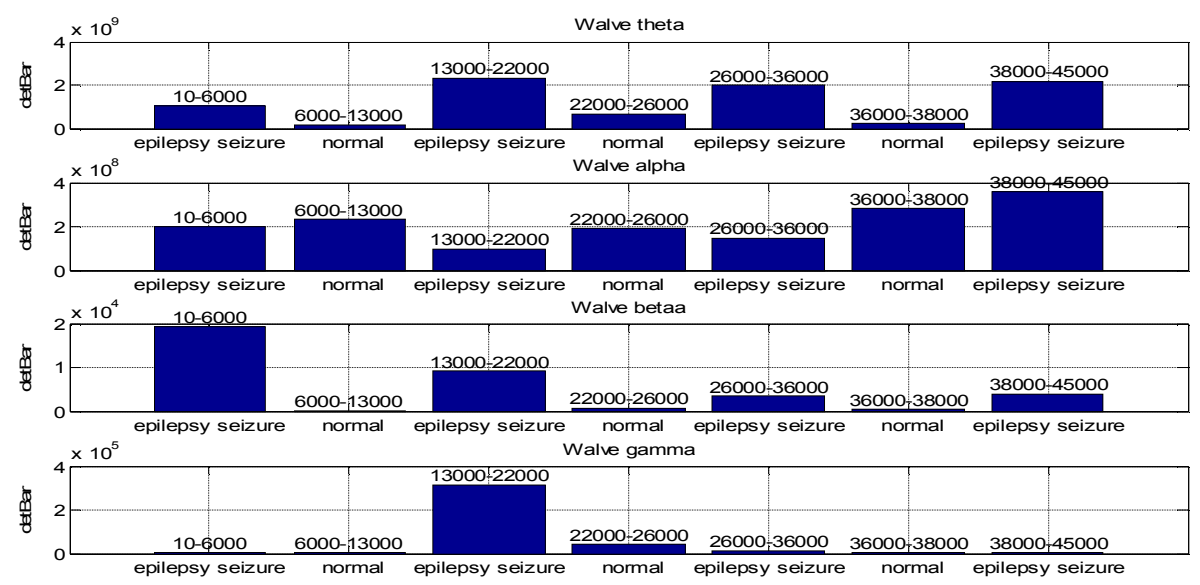

Fig. 3. Epilepsy determinant quantitative analysis.

discharging, and the beta waves are obvious. But alpha and gamma waves can't tell the two different period clearly. In conclusion, the theta waves can detect epileptic discharge period accurately, and the beta waves are less accurately than that, but the alpha and gamma waves can't able to distinguish the epileptic discharge from its stopping discharging clearly.

\section{Summary}

This paper analyzes theta, alpha, beta and gamma waves based on APSTM. The results show that when APSTM detects epileptic discharge, the theta wave is the most significant, the beta wave can only generally detect epileptic discharge, while alpha and gamma waves are ineffective. In order to analyze the rule that the four waves change with time under APSTM determinant, the quantitative analyze the seven bands determinant according to the raw EEG differential signal from two channels, the finding that the theta waves is the most accurate for APSTM detection seizure under four bands. The conclusion will have a positive significance for the study of synchronous multi-channel EEG.

\section{References}

[1] R.S. Fisher, W.V.E. Boas, W. Blume, C. Elger, P. Genton, P. Lee, J. E. Jr, Epileptic seizures and epilepsy: definitions proposed by the International League Against Epilepsy (ILAE) and the International Bureau for Epilepsy (IBE), Epilepsia. 46 (2005) 470-472.

[2] F. Mormann, K. Lehnertz, P. David, C.E. Elger, Mean phase coherence as a measure for phase synchronization and its application to the EEG of epilepsy patients, Physica D 144 (2000) 358-369.

[3] Y.C. Lai, M.G. Frei, I. Osorio, Detecting and characterizing phase synchronization in non-stationary dynamical systems, Phys. Rev. E 73 (2006).

[4] Y.C. Lai, M.G. Frei, I. Osorio, L. Huang, Characterization of Synchrony with Applications to Epileptic Brain Signals, Phys. Rev. Lett. 98 (2007).

[5] J.F. Sun, X.F. Hong, S.B. Tong, Phase Synchronization Analysis of EEG Signals: An Evaluation Based on Surrogate Tests , IEEE T. Biomed. 59 (2012) 2254-2263.

[6] M.G. Rosenblum, A.S. Pikovsky, J. Kurths, Phase synchronization of chaotic oscillators, Phys. Rev. Lett. 76 (1996) 1804-1807.

[7] A. Delorme, S. Makeig, EEGLAB: an open-source toolbox for analysis of EEG dynamics, J. Neurosci. Methods 134 (2004) 9-21. 